\title{
Mélanose essentielle ou syndrome de Laugier-Hunziker
}

\author{
Tommaso Lombardi ${ }^{1}$, Florian Combremont ${ }^{2}$, Jacky Samson ${ }^{2, *}$ \\ ${ }^{1}$ Laboratoire d'Histopathologie buccale, Ecole de Médecine dentaire, Genève, Suisse \\ ${ }^{2}$ Division de Stomatologie et Chirurgie orale, Ecole de Médecine dentaire, Genève, Suisse \\ *jacky.samson@unige.ch
}

(Reçu le le 4 octobre 2012, accepté le 2 novembre 2012)

\begin{abstract}
Résumé - La mélanose essentielle ou syndrome de Laugier-Hunziker représente un trouble de la pigmentation rare et acquis, d'étiologie inconnue, qui touche surtout les femmes caucasiennes d'âge moyen. Elle se traduit par des macules brunâtres, quelquefois noirâtres, de couleur homogène, bien limitées, intéressant le plus souvent la lévre inférieure et la région antérieure de la cavité buccale. Ces macules peuvent être associées à des macules cutanées, génitales et à des mélanonychies longitudinales.

Le cas rapporté concerne une jeune femme qui présentait une macule labiale unique, représentant sans doute la lésion initiale d'une mélanose essentielle.
\end{abstract}

\begin{abstract}
Idiopathic lenticular mucocutaneous pigmentation or Laugier-Hunziker syndrome. This pathology represents a rare acquired disorder of pigmentation of unknown etiology, which mainly affects middle-aged white women. Clinically it is characterized by several, occasionally confluent, well limited brown macules, sometimes blackish, of uniform color, affecting most often the lower lip and the anterior region of the oral cavity. These macules may be associated with cutaneous and/or genital macules, and frequently with longitudinal melanonychia.

We present the case of a young white woman who had a solitary labial macule, probably representing the initial lesion of Laugier-Hunziker syndrome.
\end{abstract}

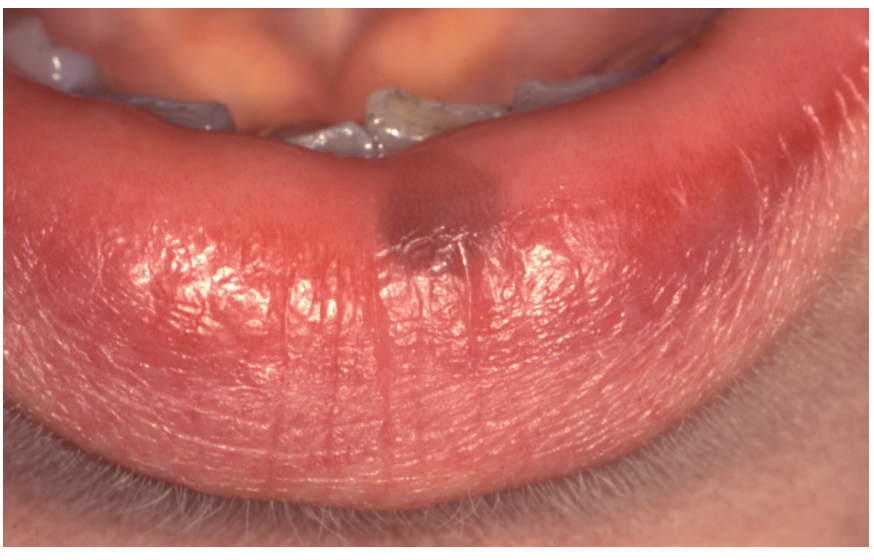

Fig. 1. Macule brunâtre isolée, d'apparition récente, intéressant la lèvre inférieure.

Fig. 1. Recently appeared solitary brownish macule involving the lower lip.

Une patiente d'origine russe, diplomate, âgée de 29 ans, est venue consulter en juin 2007 pour une macule brunâtre labiale inférieure, apparue 6 mois plus tôt. Cette macule qui siégeait dans la région para-médiane gauche, à cheval sur la muqueuse et la demi-muqueuse, avait des limites nettes et régulières et une couleur homogène; elle avait un diamétre de $6 \mathrm{~mm}$ (Fig. 1). A l'interrogatoire et à l'examen clinique, on ne retrouvait aucune autre lésion pigmentée. La patiente était en bonne santé et ne prenait aucun médicament. Elle ne fumait pas et aucune lésion n'avait précédé l'apparition de cette macule.

Bien que la lésion ne présentât aucun catactère suspect et que le diagnostic de mélanose essentielle ait été évoqué, la patiente a souhaité l'exérèse pour la confirmation du diagnostic et surtout en raison du préjudice esthétique. L'examen histopathologique (Fig. 2) a montré qu'il existait par endroits une discrète hyperpigmentation de l'assise basale avec migration du pigment mélanique dans le chorion superficiel où il est libre ou repris par des mélanophages. Il n'existait aucune prolifération mélanocytaire. L'aspect était donc compatible avec celui d'une mélanose essentielle. 


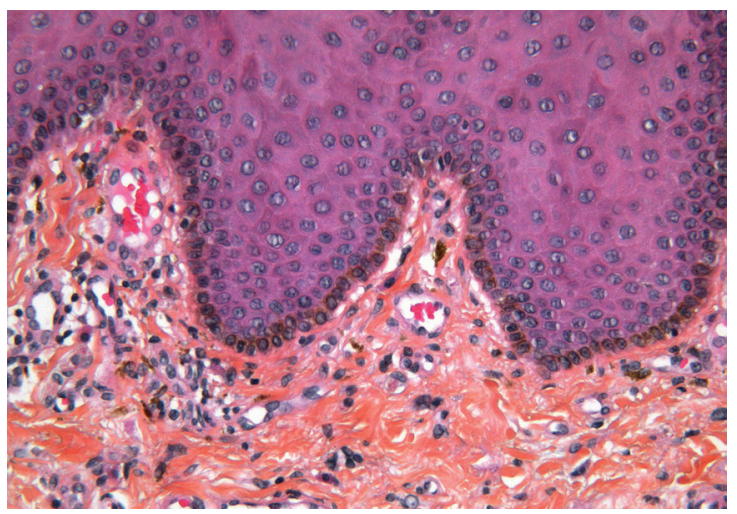

Fig. 2. Hyperpigmentation de la couche basale avec incontinence du pigment mélanique, parfois repris par des mélanophages (HES, x20). Fig. 2. Basal cells hyperpigmentation with loss of melanin pigment into the papillary chorion where it is taken up by scattered melanophages (HES, x20).

La description princeps de la mélanose essentielle a été réalisée en 1970 par Laugier et Hunziker alors responsable du Service de Dermatologie de l'Hôpital cantonal de Genève [3]. Cette affection, dont l'étiologie reste non élucidée, a été rapidement plus connue sous le terme de syndrome de Laugier-Hunziker ; elle n'est jamais associée à un autre syndrome ou à une affection systémique [5]. Cliniquement, elle se traduit par des macules brunâtres ou noirâtres, de quelques $\mathrm{mm}$ de diamétre, de couleur homogène, avec des limites nettes et régulières, intéressant principalement la muqueuse de la partie antérieure de la cavité buccale, surtout la lévre inférieure. Ces macules qui n'apparaissent pas toutes simultanément et qui s'observent surtout chez des femmes caucasiennes d'âge moyen [4], sont accompagnées dans 50 à $60 \%$ des cas par des macules cutanées, génitales, anales, ou par des mélanonychies longitudinales, exceptionnellement par des macules conjonctivales ou oesophagiennes [2]. Ces lésions ne présentent aucun potentiel de transformation maligne. La dermatosopie est peu contributive car l'aspect des lésions est très variable [2]. Pour le diagnostic différentiel, on peut évoquer le syndrome de Peutz-Jeghers, la maladie
d'Addison, un mélanoacanthome [1] ou un mélanome [4] mais le contexte ou l'aspect microscopique est bien différent.

A l'examen histopathologique, on retrouve une hyperpigmentation mélanique dans la couche basale de l'épithélium qui est souvent acanthosique. Il n'y a pas d'anomalies dans le nombre, la morphologie et la distribution des mélanocytes mais il existe une incontinence pigmentaire superficielle et le pigment est parfois repris par des mélanophages dans le chorion superficiel. Cet aspect n'est pas spécifique et c'est son interprétation avec les données cliniques qui permet de confirmer le diagnostic. Dans le cas rapporté, l'aspect de la macule faisait évoquer d'emblée une mélanose essentielle mais, face à une macule isolée, on manqait d'arguments pour confirmer le diagnostic. Il avait été prévu de revoir la patiente pour suivre l'évolution dans l'attente de l'apparition de nouvelles macules qui auraient permis d'établir avec certitude le diagnostic mais ceci n'a pas été possible en raison du changement d'affectation de la patiente.

Conflits d'intérêt : aucun

\section{Références}

1. Galindo-Moreno P, Padial-Molina M, Gomez-Morales M, Aneiros-Fernandez J, Mesa F, O'Valle F. Multifocal oral melanoacanthoma and melanotic macula in a patient after dental implant surgery. JADA 2011;142:817-24.

2. Ko J-H, Shih Y-C, Chiu C-S, Chuang Y-H. Dermatoscopic features in Laugier-Hunziker syndrome. J Dermatol 2011;38:87-90.

3. Laugier P, Hunziker N. Pigmentation mélanique essentielle de la muqueuse jugale et des lévres. Arch Belg Dermatol Syphilol 1970;26:391-9.

4. Shen Z-Y, Liu W, Bao Z-X, Zhou Z-T, Wang L-Z. Oral melanotic macule and primary oral malignant melanoma: epidemiology, location involved, and clinical implications. Oral Surg Oral Med Oral Pathol Oral Radiol Endod 2011;112:e21-5.

5. Wondratsch H, Feldmann R, Steiner A, Breier F. LaugierHunziker syndrome in a patient with pancreatic cancer. Case Rep Dermatol 2012;4:174-6. 